Supporting Information

\title{
Europium Ionic Liquid Grafted Covalent Organic Framework with Dual Luminescence Emissions as Sensitive and Selective Acetone Sensor
}

\author{
Hongyu Zuo, ${ }^{\dagger}$ Ying Li ${ }^{*},+$ Yaozu Liao ${ }^{*},+$
}

[†] School of Materials Science and Engineering, University of Shanghai for Science and

Technology, Shanghai 200093, China

[¥] State Key Laboratory for Modification of Chemical Fibers and Polymer Materials, College of Materials Science and Engineering, Donghua University, Shanghai 201620, China

Corresponding author: liying@usst.edu.cn; yzliao@dhu.edu.cn

\section{Experimental sections}

Chemicals. 2,5-dihydroxyterephthalaldehyde (Dha, 95\%), 1,3,5-tris(4-aminophenyl)benzene (Tab, 93\%), $n$-butyl alcohol ( $n$-BuOH, 99\%), o-dichlorobenzene (o-DCB, 98\%), Europium chloride $\left(\mathrm{EuCl}_{3} \cdot 6 \mathrm{H}_{2} \mathrm{O}, 99.9 \%\right)$ and 2-thenoyltrifluoroacetone (TTA, 98\%) were purchased from Aladdin Chemistry (Shanghai, China). Potassium carbonate $\left(\mathrm{K}_{2} \mathrm{CO}_{3}, 99 \%\right), \mathrm{N}$, N'-dimethylformamide (DMF, 99\%), acetonitrile, and 1,2-dibromoethane were obtained from Guoyao Factory (Shanghai, China). All of the other chemical reagents were of analytical grade.

Synthesis of (2-bromoethyl)triethylammonium bromide $\left(\left[\mathrm{BrEt}_{4} \mathrm{~N}\right] \mathrm{Br}\right)$. A solution of triethylamine (1.01 g, $9.9 \mathrm{mmol})$ and 1,2-dibromoethane $(9.28 \mathrm{~g}, 49.4 \mathrm{mmol}) \mathrm{in}_{\mathrm{CH}} \mathrm{CN}(50 \mathrm{~mL})$ was refluxed for $4 \mathrm{~h}$. Rotary distillation was used to remove residual dibromoethane and acetonitrile. The obtained precipitate was washed with ethyl acetate, then isolated by filtration 
and finally dried at $80{ }^{\circ} \mathrm{C}$ under vacuum to give (2-bromoethyl)triethylammonium bromide $\left(\left[\mathrm{BrEt}_{4} \mathrm{~N}\right] \mathrm{Br}\right)\left(1.2 \mathrm{~g}, 41 \%\right.$ yield). ${ }^{1} \mathrm{H} \mathrm{NMR}\left(\mathrm{CDCl}_{3}\right): \delta(\mathrm{ppm})=3.91(\mathrm{t}, 2 \mathrm{H}), 3.80(\mathrm{t}, 2 \mathrm{H}), 3.68(\mathrm{q}$, $6 \mathrm{H}), 1.46(\mathrm{~s}, 9 \mathrm{H}) ;{ }^{13} \mathrm{C} \mathrm{NMR}\left(\mathrm{CDCl}_{3}\right): \delta(\mathrm{ppm})=57.86,54.20,21.77,8.36$. The ${ }^{1} \mathrm{H}$ NMR and ${ }^{13} \mathrm{C}$ NMR spectra of $\left[\mathrm{BrEt}_{4} \mathrm{~N}\right] \mathrm{Br}$ can be seen in the appendix.

Synthesis of DhaTab-COF. Tab (140.58 mg, $0.4 \mathrm{mmol})$ and Dha (99.68 mg, $0.6 \mathrm{mmol})$ were weighed into a glass flask $(c a .50 \mathrm{~mL})$, which were suspended in $n-\mathrm{BuOH}(10 \mathrm{~mL})$ and $o$-DCB $(10 \mathrm{~mL})$ with $1 \mathrm{~mL}$ of $6 \mathrm{M} \mathrm{AcOH}$. After sonicated solution for $10 \mathrm{~min}$ and reflowed at $120^{\circ} \mathrm{C}$ for 3 days under a nitrogen atmosphere. The orange precipitate was collected by centrifugation, then extracted via a dropping funnel with THF for $12 \mathrm{~h}$. The powder product with a yield of $90 \%$ yield was transferred to a clean vial and dried at $100^{\circ} \mathrm{C}$ for $12 \mathrm{~h}$ under vacuum.

Synthesis of DhaTab-COF-IL. DhaTab-COF (100 mg), [BrEt $\left.t_{4} \mathrm{~N}\right] \mathrm{Br}(100 \mathrm{mg})$, and $\mathrm{K}_{2} \mathrm{CO}_{3}(70$ $\mathrm{mg}$ ) in DMF (35 mL) was stirred under reflux for $24 \mathrm{~h}$. After cooling, the precipitate was collected by filtration, washed with deionized water, ethanol and acetone, then dried at $100{ }^{\circ} \mathrm{C}$ under vacuum for $12 \mathrm{~h}$ to give DhaTab-COF-IL quantitatively as a dark red solid with a yield of $50 \%$.

Synthesis of DhaTab-COF-EuIL. TTA $(0.266 \mathrm{~g}, 1.2 \mathrm{mmol})$ and $\mathrm{NaOH}(1.2 \mathrm{mmol}, 0.048 \mathrm{~g})$ were immersed in ethanol $(15 \mathrm{ml})$, then stirred under reflux for $1 \mathrm{~h}$. Then $\mathrm{EuCl}_{3} \cdot 6 \mathrm{H}_{2} \mathrm{O}(0.11 \mathrm{~g}$, $0.3 \mathrm{mmol}$ ) was added to react $24 \mathrm{~h}$. After cooling, the precipitate was collected by filtration, washed with ethanol, and then dried at $70{ }^{\circ} \mathrm{C}$ under vacuum for $12 \mathrm{~h}$ to obtain DhaTab-COF-EuIL as dark red solid with a yield of $45 \%$.

Characterization and Measurements. Fourier transform infrared (FT-IR) spectra were taken on a Nicolet 670 spectrometer. Solid state ${ }^{13} \mathrm{C}$ cross-polarization magic angle spinning nuclear magnetic resonance (CP/MAS NMR) spectra were taken on an AVANCE400 spectrometer. Powder X-ray diffraction (XRD) patterns were obtained on a Bruker D8 Advance diffractometer (40 kV, 30Ma) using $\mathrm{Cu} \mathrm{K \alpha}$ radiation $\left(2 \theta=2-40^{\circ}\right)$. X-ray photoelectron spectra (XPS) were obtained on a PHI5000 1 Versa probe-II multifunctional 2 scanning and imaging photoelectron spectrometer (Japan) equipped with an $\mathrm{Al} \mathrm{K \alpha} \mathrm{X}$-ray source. Thermal gravimetric analysis (TGA) was carried out on a TGA Q500 apparatus under a nitrogen atmosphere in the temperature range 30-800 ${ }^{\circ} \mathrm{C}$ (heating rate $10{ }^{\circ} \mathrm{C} \mathrm{min}^{-1}$ ). Scanning electron microscope (SEM) images were 
obtained on a HITACHI S-4800 and transmission electron microscope (TEM) images were obtained on a JEM-2100 TEM microscope.

Structural Simulation. Density functional tight-binding (DFTB) method was applied in order to simulate the structure of these COFs and the unit cell parameters. The experimental PXRD patterns are agreeable with the simulated patterns of some near-eclipsed stacking models (Figure $\mathrm{S} 2$ and S3). Hence, we propose structures close to hexagonal space group for DhaTab-COFs by comparing the experimental and simulated PXRD patterns. Refinements of PXRD pattern were done using Reflex module of Material Studio software.

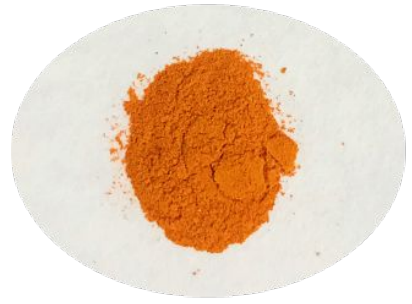

DhaTab-COF

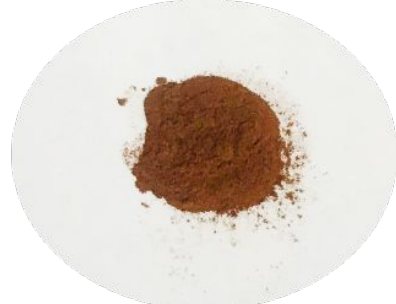

DhaTab-COF-IL

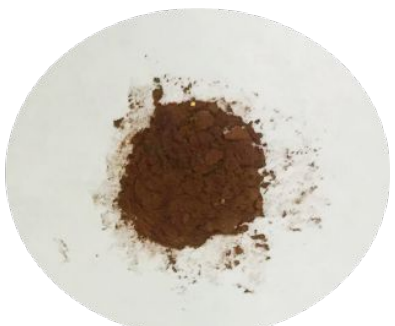

DhaTab-COF-EulL

Figure S1 Appearances of DhaTab-COF, DhaTab-COF-IL, and DhaTab-COF-EuIL.
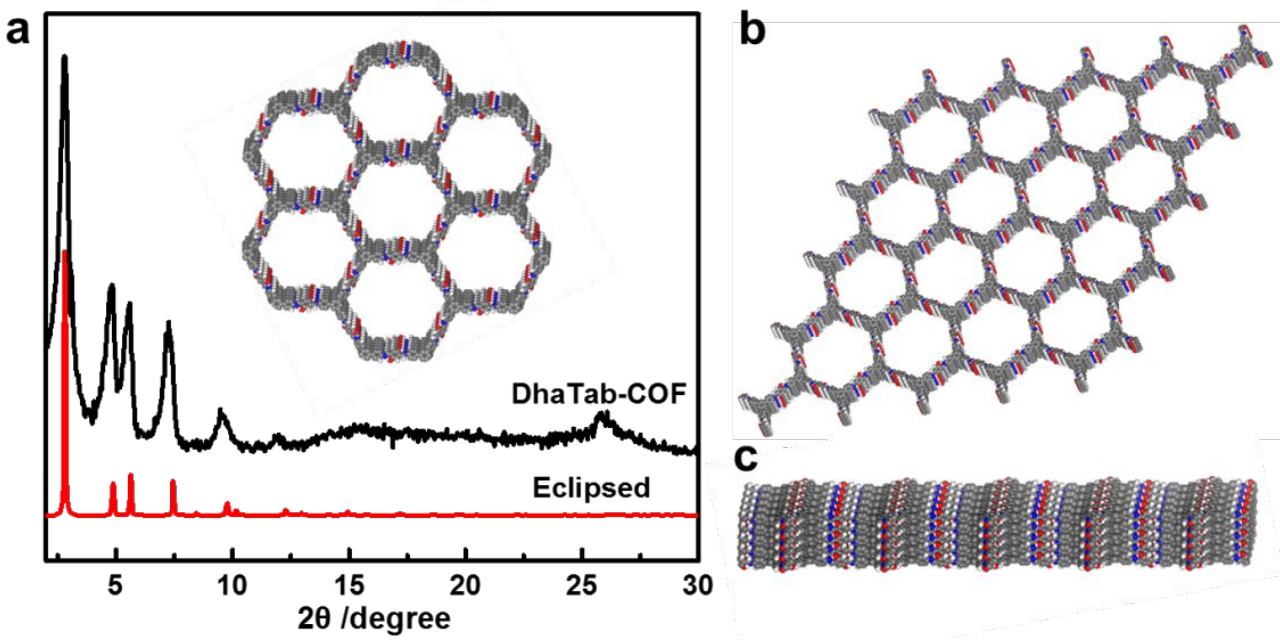

Figure S2 (a) The experimental PXRD pattern of DhaTab-COF (black) compared with simulated eclipsed (red), (b) the eclipsed stacking model and (c) stacking diagram of DhaTab-COF. Inset in Figure S2a showing top view of the eclipsed stacking model. 

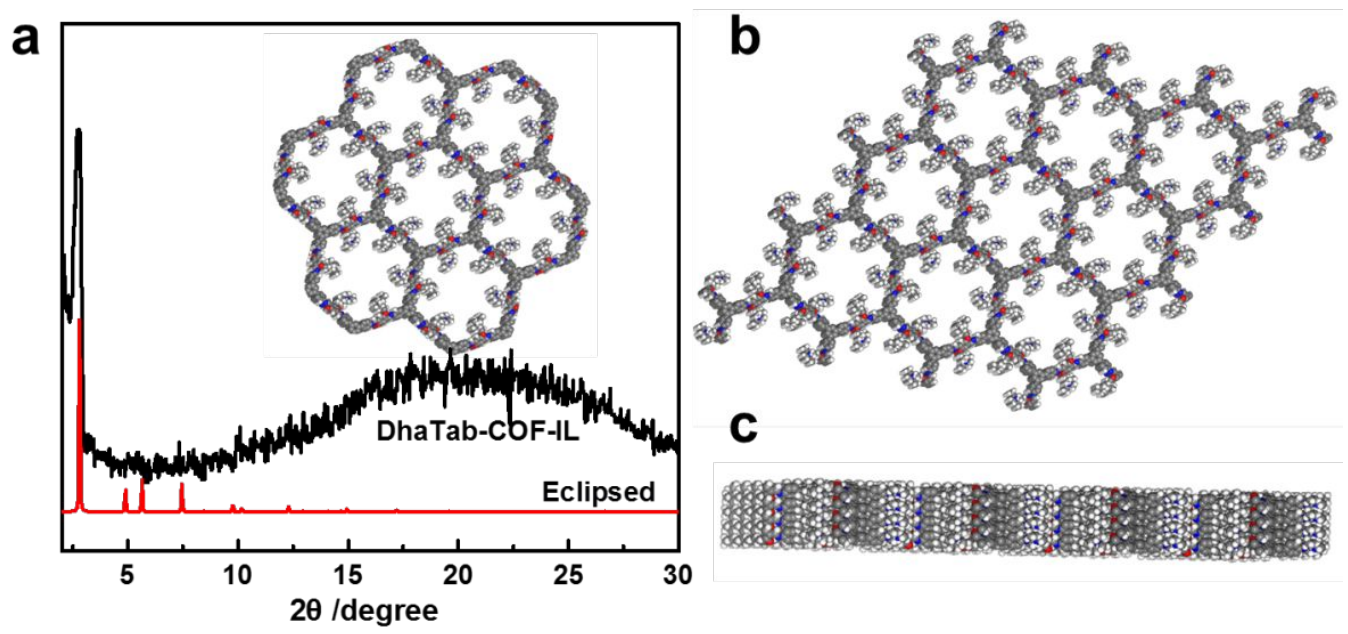

Figure S3 (a) The experimental PXRD pattern of DhaTab-COF-IL (black) compared with simulated eclipsed (red), (b) the eclipsed stacking model and (c) stacking diagram of DhaTab-COF-IL. Inset in Figure S3a showing top view of the eclipsed stacking model.

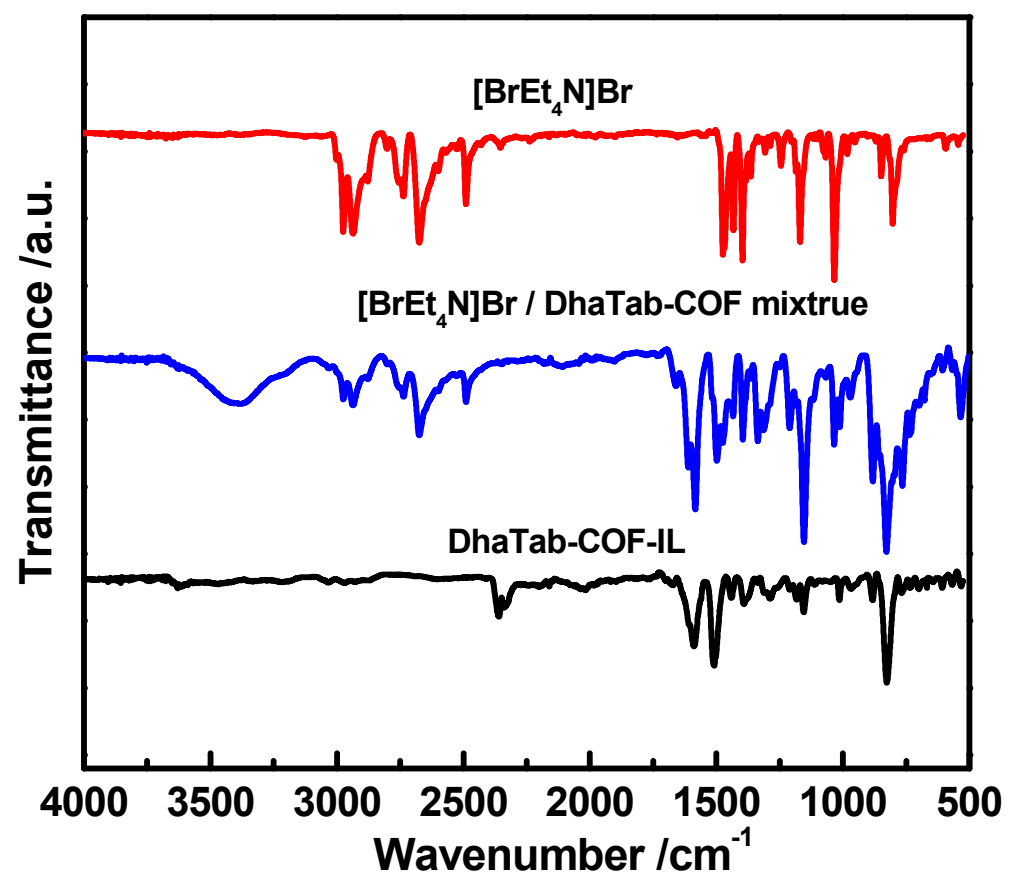

Figure S4 FT-IR spectra of $\left[\mathrm{BrEt}_{4} \mathrm{~N}\right] \mathrm{Br}, \mathrm{BrEt}_{4} \mathrm{NBr} /$ DhaTab-COF mixture and DhaTab-COF-IL. 


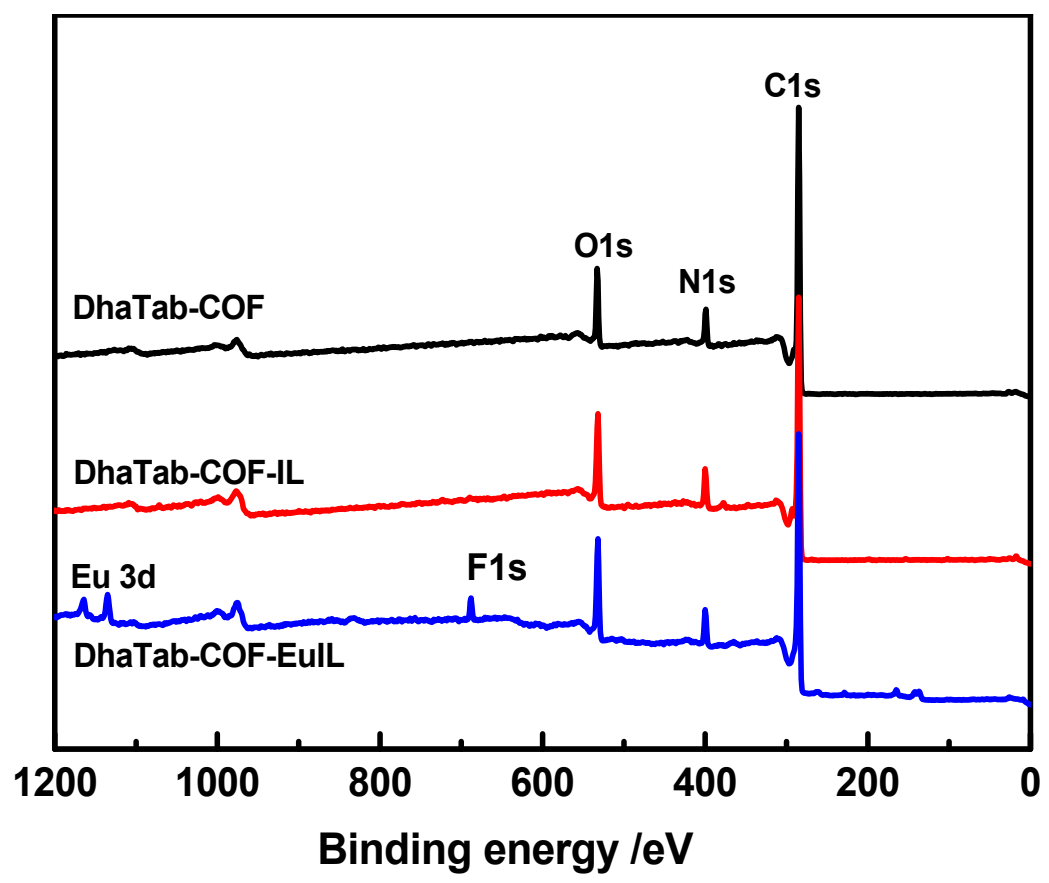

Figure S5 Full survey XPS spectra of DhaTab-COF, DhaTab-COF-IL, and DhaTab-COF-EuIL.

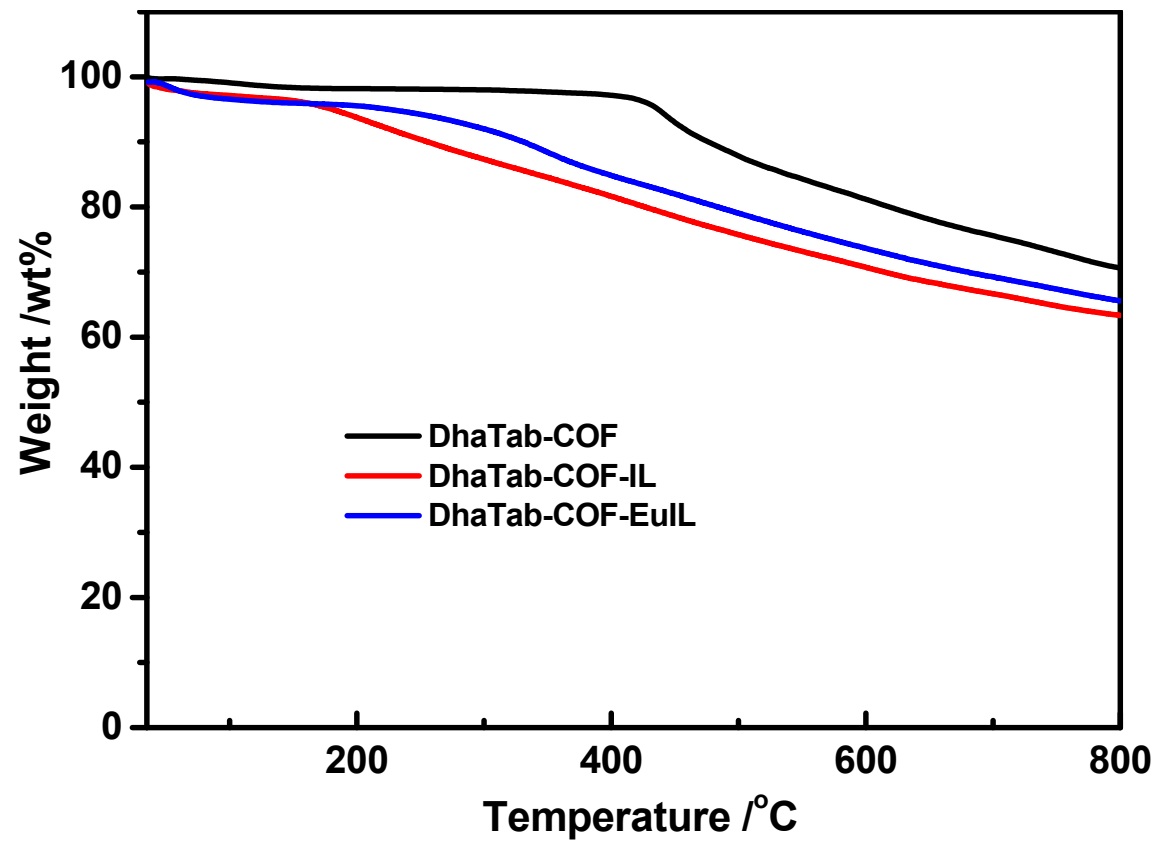

Figure S6 TGA of DhaTab-COF, DhaTab-COF-IL, DhaTab-COF-EuIL. 


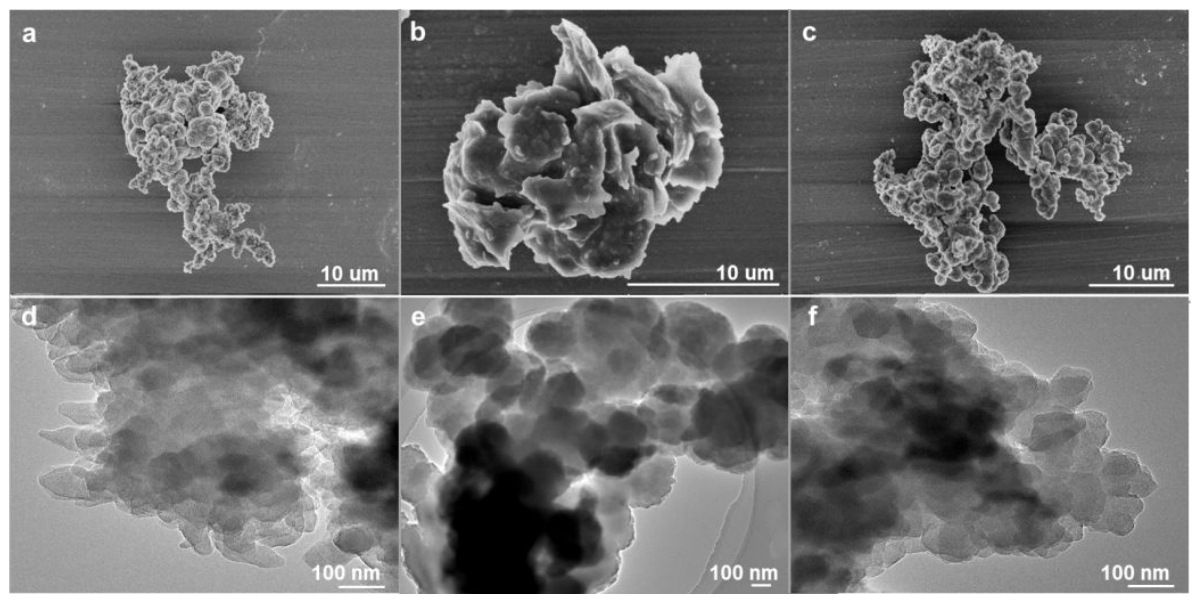

Figure S7 SEM and TEM images of (a,d) DhaTab-COF, (b,e) DhaTab-COF-IL, and (c,f) DhaTab-COF-EuIL.

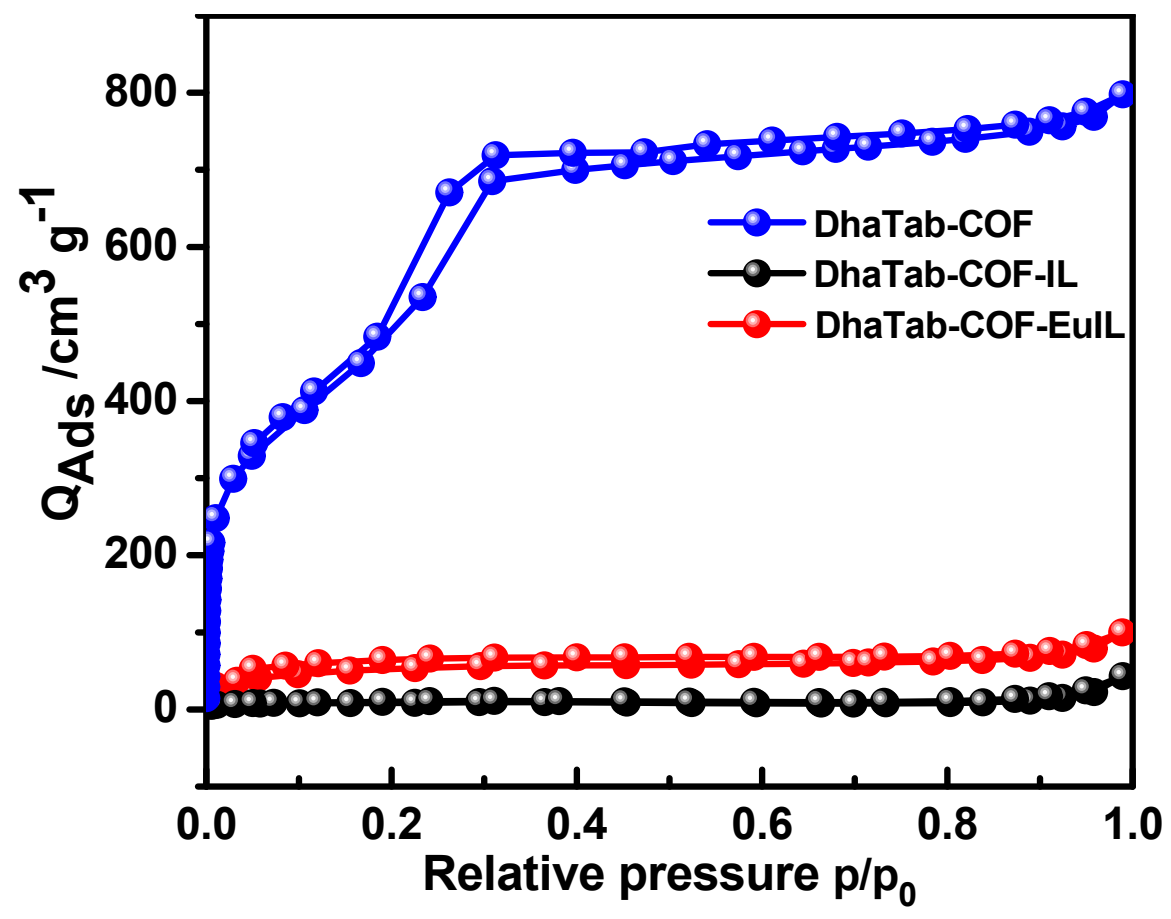

Figure $\mathbf{S 8} \mathrm{N}_{2}$ adsorption/desorption isotherms of DhaTab-COF, DhaTab-COF-IL, DhaTab-COF-EuIL 

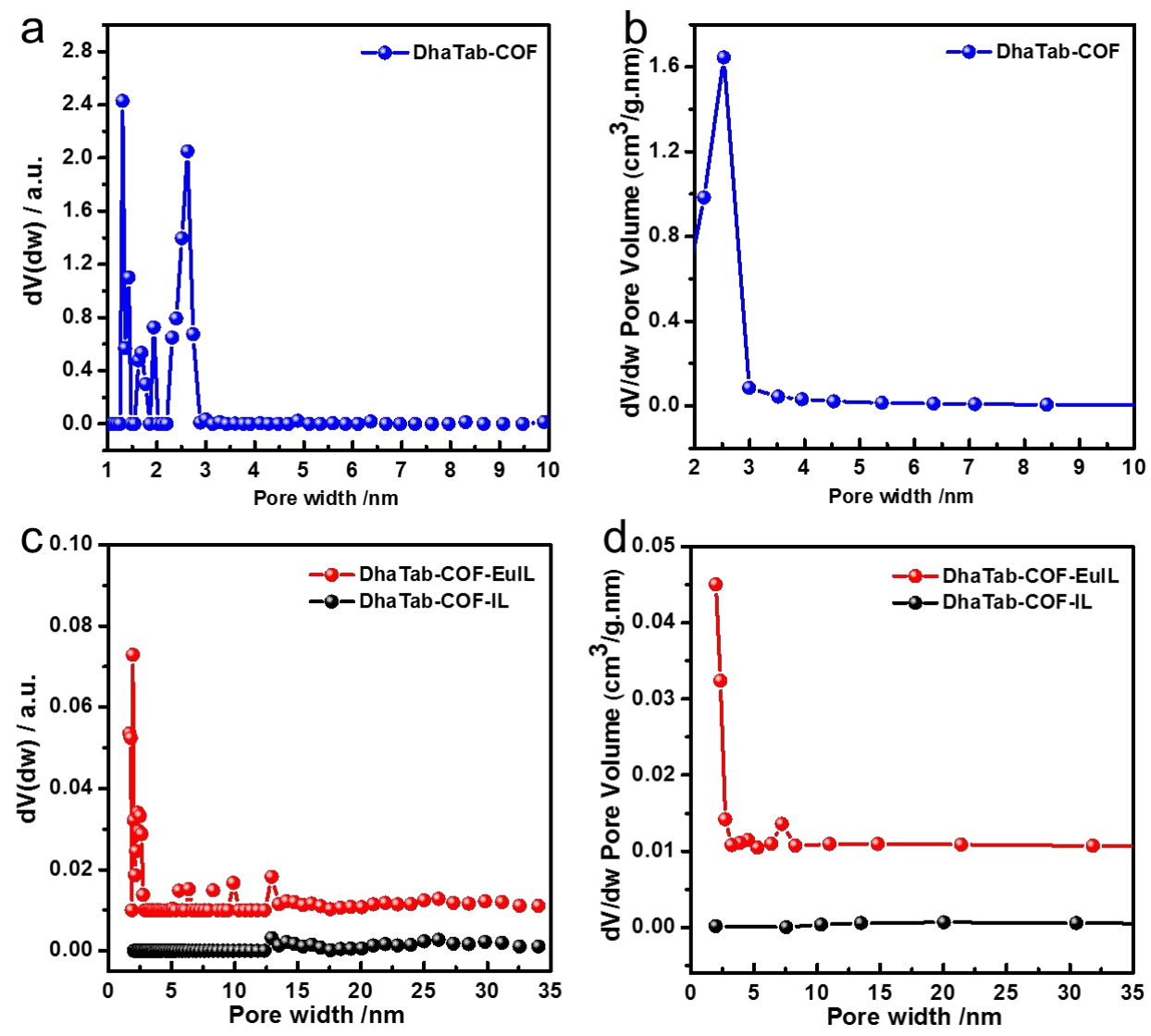

Figure S9 $(a, c)$ NLDFT and (b,d) BJH pore size distributions of DhaTab-COF, DhaTab-COF-IL, and DhaTab-COF-EuIL.

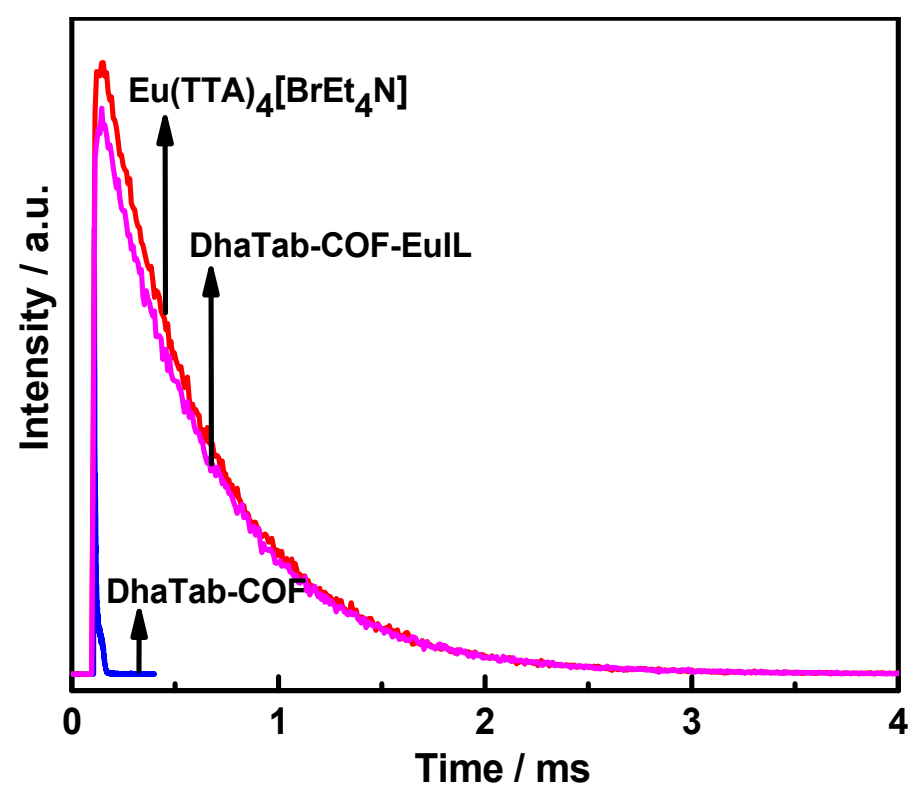


Figure S10 Decay curves of DhaTab-COF, DhaTab-COF-EuIL, and Eu(TTA) ${ }_{4}\left[\mathrm{BrEt}_{4} \mathrm{~N}\right]$.

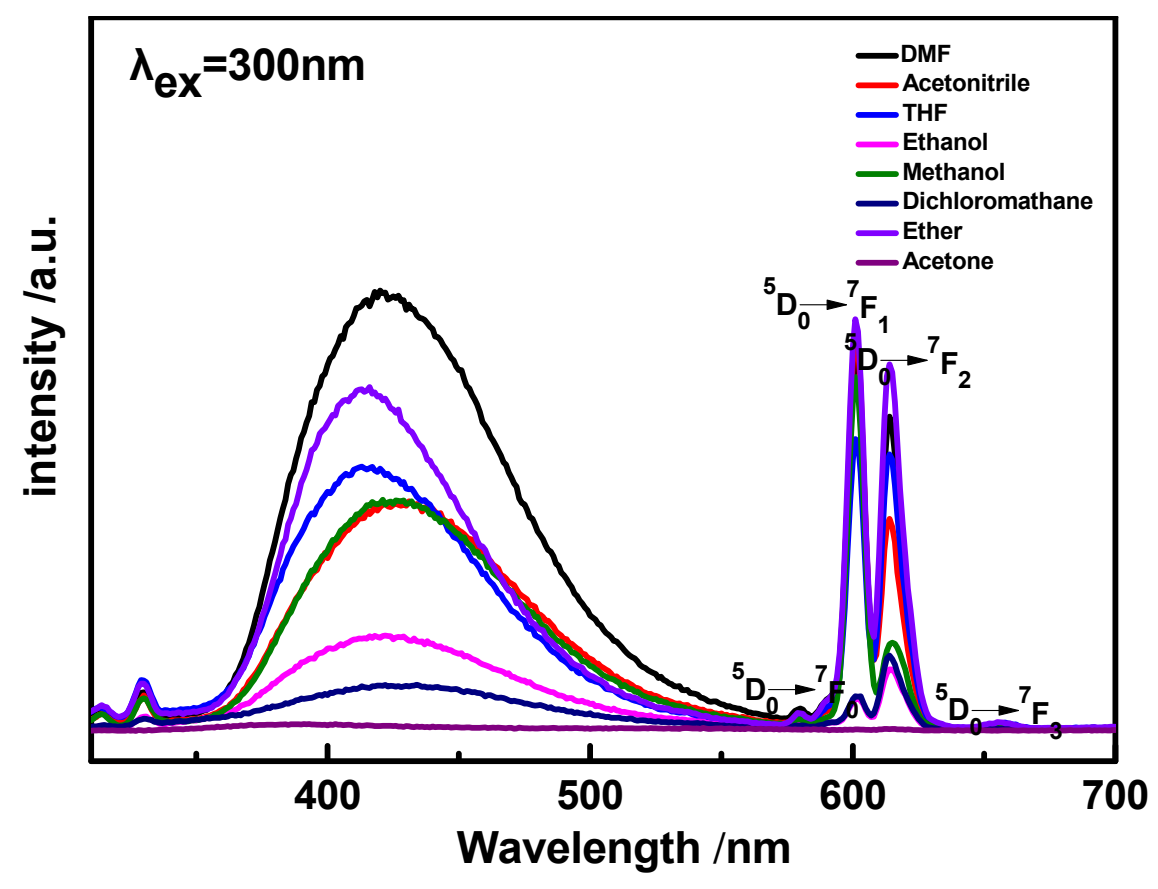

Figure S11 Luminescent spectra of DhaTab-COF-EuIL dispersed in different solvents.

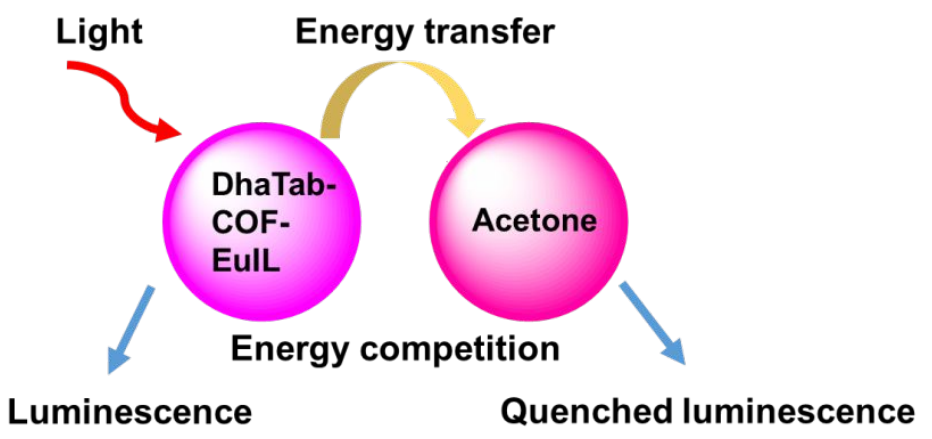

Figure S12 The luminescence quenching mechanism of DhaTab-COF-EuIL in presence of acetone. 


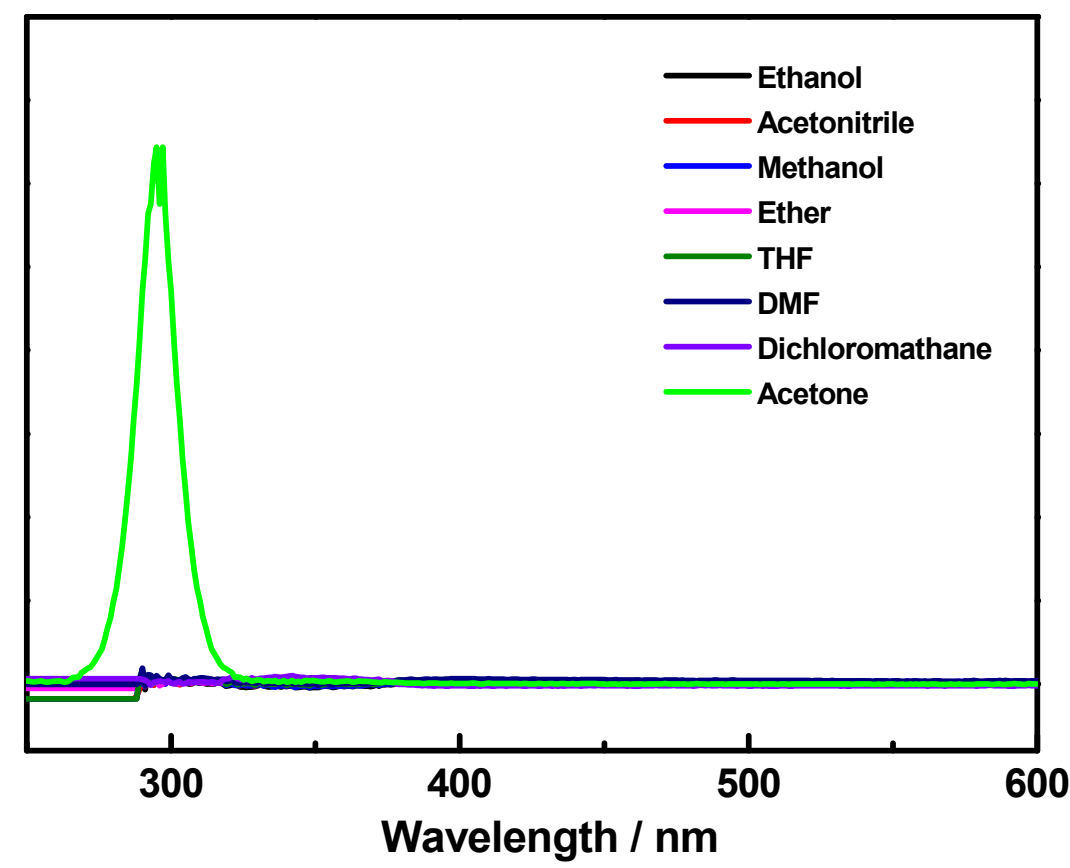

Figure S13 UV-vis spectra of ethanol, acetonitrile, methanol, ether, THF, DMF, dichloromethane.

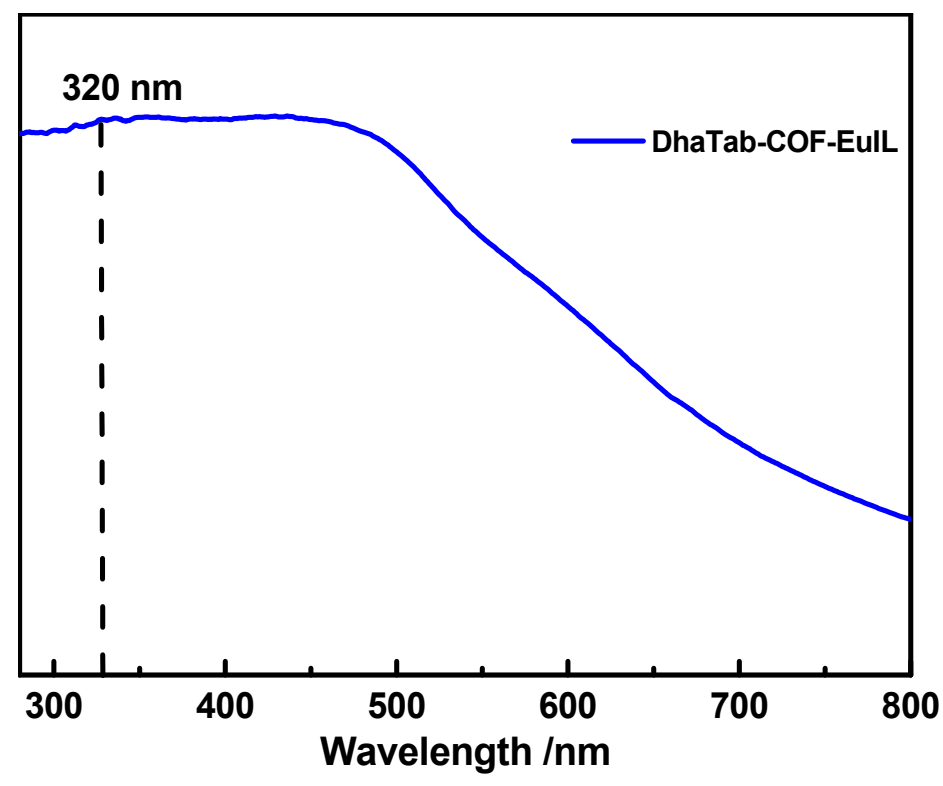

Figure S14 UV-vis spectra of DhaTab-COF-EuIL. 
Table S1 Luminescence lifetimes of DhaTab-COF, DhaTab-COF-EuIL, Eu(TTA) ${ }_{4}\left[\mathrm{BrEt}_{4} \mathrm{~N}\right]$

\begin{tabular}{cc}
\hline Compounds & Luminescence lifetime (ms) \\
\hline DhaTab-COF & 0.136 \\
DhaTab-COF-EuIL & 0.529 \\
Eu(TTA $)_{4}\left[\mathrm{BrEt}_{4} \mathrm{~N}\right]$ & 0.656 \\
\hline
\end{tabular}

Table S2 The comparison of limit of detection for acetone using different sensors

\begin{tabular}{|c|c|c|}
\hline Sensors & $\begin{array}{c}\text { Limit of detection } \\
(\text { vol\%) }\end{array}$ & References \\
\hline DhaTab-COF-EuIL & 1.0 & This work \\
\hline$\{[\mathrm{Tb}(\mathrm{FDA}) 1.5(\mathrm{DMF})] \cdot \mathrm{DMF}\} \mathrm{n}$ & 14 & 43 \\
\hline$\left[\mathrm{Me}_{2} \mathrm{NH}_{2}\right]_{2}\left[(\mathrm{Eu})_{2}(\mathrm{ofdp})_{2}(\mathrm{DMF})\left(\mathrm{H}_{2} \mathrm{O}\right)\right]$ & 7.4 & 44 \\
\hline \multicolumn{3}{|l|}{$\cdot 7 \mathrm{H}_{2} \mathrm{O} \cdot \mathrm{DMF}$} \\
\hline $\mathrm{Yb}(\mathrm{BPT})\left(\mathrm{H}_{2} \mathrm{O}\right) \cdot(\mathrm{DMF}) 1.5 \cdot\left(\mathrm{H}_{2} \mathrm{O}\right)$ & 5 & 45 \\
\hline$\left[\mathrm{Eu}_{4}(\mathrm{BPT})_{4}(\mathrm{DMF})_{2}\left(\mathrm{H}_{2} \mathrm{O}\right)_{8}\right]$ & 5 & 46 \\
\hline $\mathrm{CB}[6]$-based supramolecular & 5 & 47 \\
\hline$\left[\mathrm{Eu}_{2}\left(\mathrm{u}_{2}-\mathrm{pzdc}\right)\left(\mathrm{m}_{4}-\mathrm{pzdc}\right)\left(\mathrm{m}_{2}-\mathrm{ux}\right)\left(\mathrm{H}_{2} \mathrm{O}\right)_{4}\right] \cdot 8 \mathrm{H}_{2} \mathrm{O}$ & 5.75 & 48 \\
\hline $\mathrm{Zn}-\mathrm{PDC} / \mathrm{Tb}^{3+}$ & 10 & 49 \\
\hline
\end{tabular}




\section{Appendix}

The ${ }^{1} \mathrm{H}$ NMR and ${ }^{13} \mathrm{C}$ NMR spectra of (2-bromoethyl)triethylammonium bromide $\left(\left[\mathrm{BrEt}_{4} \mathrm{~N}\right] \mathrm{Br}\right)$

${ }^{1} \mathrm{H} \mathrm{NMR}$ in $\mathrm{CDCl}_{3}$

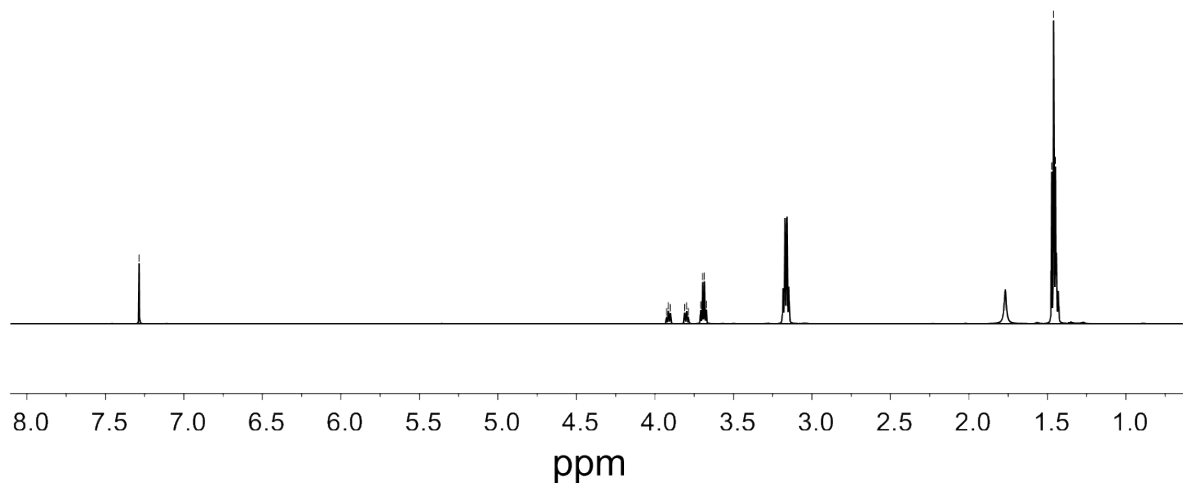

${ }^{13} \mathrm{C} \mathrm{NMR} \mathrm{in} \mathrm{CDCl}_{3}$ 
ิㅡ으용

ペ゚゚

$\infty$ ิ

is if

$\begin{array}{cc}N & 0 \\ 亡 & \infty \\ 亡 & \infty\end{array}$

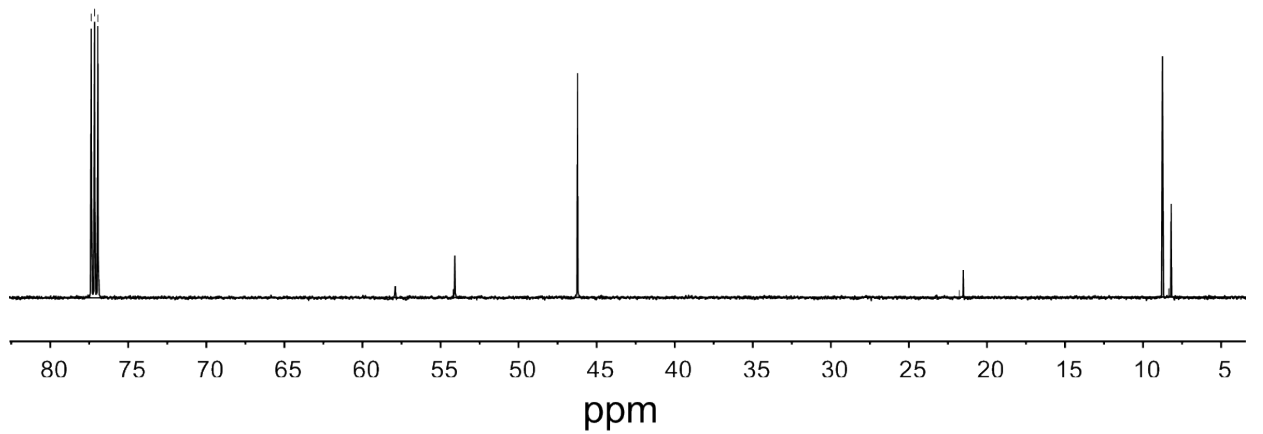

S-12 